\title{
Células Cebadas y sus Patrones de Expresión del Receptor c-Kit (CD117) como Probables Indicadores de Metástasis en el Adenocarcinoma de Mama en Humanos
}

\author{
Mast Cells and Expression Patterns of c-Kit (CD117) Receptor as \\ Probable Indicators of Metastases in Human Breast Adenocarcinoma
}

Oscar Mario Hernández-Alvidrez*; Adolfo Soto-Domínguez **; Eduardo Alfredo González-Murillo; Edmundo Erbey Castelán-Maldonado*; David García-Garza*** \& Rubén García-Garza**

HERNÁNDEZ-ALVIDREZ, O. M.; SOTO-DOMÍNGUEZ, A.; GONZÁLEZ-MURILLO, E. A.; CASTELÁN-MALDONADO, E. E.; GARCÍA-GARZA, D. \& GARCÍA-GARZA, R. Células cebadas y sus patrones de expresión del receptor c-Kit (CD117) como probables indicadores de metástasis en el adenocarcinoma de mama en humanos. Int. J. Morphol., 31(2):600-605, 2013.

RESUMEN: El adenocarcinoma de mama es la segunda neoplasia maligna más común en mujeres, solo después del cáncer de pulmón. Este tumor se clasifica histopatológicamente en bien, moderado y poco diferenciado. Además de esta clasificación, los análisis actuales utilizan inmunohistoquímica para identificar receptores hormonales para estrógenos y progesterona (RH) y HER2/neu, esta información permite hacer un pronóstico de respuesta al tratamiento y tiempo de sobrevida. Las células cebadas se han asociado a un mal pronóstico, ya que participan en la angiogénesis y el crecimiento tumoral favoreciendo la metástasis. Esto disminuye la esperanza de vida. También las células cebadas expresan en la membrana plasmática el receptor c-Kit (CD117) que se ha asociado a proliferación celular. En este estudio, se evaluó si existen variaciones en los patrones de expresión de c-Kit en las células cebadas presentes en muestras de adenocarcinoma de mama; y si los distintos patrones del receptor se asocian a la presencia de metástasis linfática. Se utilizaron muestras de adenocarcinoma moderadamente diferenciado, y se analizaron empleando métodos histológicos, histoquímicos e inmunohistoquímicos. Los grupos se clasificaron de acuerdo a su positividad a RH y HER2/neu, y se subclasificaron de acuerdo a la presencia o no de metástasis. Además se realizó un análisis morfométrico de células cebadas y los patrones de expresión de c-Kit. Los grupos con metástasis mostraron mayor cantidad de células cebadas comparados con el grupo control, y su grupo correspondiente sin metástasis. Las células cebadas con patrones de expresión 1 y 3 fueron más abundantes en los grupos con metástasis. En los grupos sin metástasis predominaron las células cebadas con el patrón 2. Los resultados sugieren que el número de células cebadas y los patrones de expresión de c-Kit, podrían ser empleados como indicadores probables, más no definitivos de la presencia de metástasis linfática.

PALABRAS CLAVE: Adenocarcinoma de mama; Metástasis; Células cebadas.

\section{INTRODUCCIÓN}

El adenocarcinoma de mama es la segunda neoplasia maligna más común en mujeres después del cáncer de pulmón. Se presenta más frecuentemente en el cuadrante superolateral de la glándula mamaria izquierda (50\%) (Dixon et al., 1985).

El sistema de graduación histopatológica más utilizado es la escala de Scarff Bloom Richardson (SBR), esta escala se basa en la evaluación de varias características celulares, tales como: la forma del núcleo, la disposición celular y la tasa de mitosis. A cada una de estas características se les asigna un puntaje, y la suma de los puntajes permite clasificar el adenocarcinoma en bien, moderado y poco diferenciado. La clasificación del adenocarcinoma mamario está íntimamente relacionada con la sobrevida del paciente (Amat et al., 2002).

Además de la escala de SBR, los análisis actuales del adenocarcinoma de mama utilizan métodos inmunohistoquímicos para detectar receptores hormonales de estrógenos y progesterona $(\mathrm{RH})$, y el receptor del factor de

\footnotetext{
* Departamento de Anatomía Patológica. Unidad Médica de Alta Especialidad No. 25. IMSS Monterrey, N.L. México.

** Departamento de Histología, Facultad de Medicina, Universidad Autónoma de Nuevo León. Monterrey, N.L. México.

**** Laboratorio de Síntesis de Nanoestructuras y Nanomateriales. Facultad de Ciencias Fisicomatemáticas. Universidad Autónoma de Nuevo León. Monterrey, N.L. México.
} 
HERNÁNDEZ-ALVIDREZ, O. M.; SOTO-DOMÍNGUEZ, A.; GONZÁLEZ-MURILLO, E. A.; CASTELÁN-MALDONADO, E. E.; GARCÍA-GARZA, D. \& GARCÍA-GARZA, R. Células cebadas y sus patrones de expresión del receptor c-Kit (CD117) como probables indicadores de metástasis en el adenocarcinoma de mama en humanos. Int. J. Morphol., 31(2):600-605, 2013.

crecimiento epidérmico humano tipo-2 (Her2/neu) (Harvey et al., 1999; Mohisin et al., 2004). La detección de estos receptores permite hacer un pronóstico sobre la evolución de la enfermedad (Allred et al., 1998; Elledge et al., 2000; Bilous et al., 2003), así como una probable respuesta al tratamiento (Elston \& Ellis, 1991; Philips et al., 2007; García-Closas et al., 2008).

Por otro lado, las células cebadas son un tipo celular derivado de precursores hematopoyéticos que se encuentran en la médula ósea (Kitamura et al., 1987). Las células cebadas son ampliamente conocidas por su participación en las reacciones anafilácticas (Metcalfe et al., 1997). Sin embargo, diversos estudios han reportado un incremento de estas células en tejido de adenocarcinoma de mama (Edwin et al., 1985; Ashish et al., 2008), así como su participación favoreciendo la angiogénesis y el crecimiento del tumor (Norrby \& \& Sörbo, 1992).

El receptor de membrana c-Kit (CD117), se expresa normalmente en las células cebadas, además de otras poblaciones celulares (Columbo et al., 1992). El patrón de inmunoreacción del receptor c-Kit en las células cebadas generalmente se observa en la membrana plasmática, sin embargo se ha descrito que cuando la positividad se observa de forma difusa en el citoplasma existe una mayor tasa de proliferación celular (Webster et al., 2004).

A la fecha no existen estudios que evalúen los patrones de expresión del receptor c-Kit en las células cebadas presentes en muestras de adenocarcinoma de mama, y si el predominio de alguno de los patrones se asocia a la presencia de metástasis.

\section{MATERIAL Y MÉTODO}

Material Biológico. Se utilizaron 50 muestras de mama incluidas en bloques de parafina y previamente diagnosticadas como adenocarcinoma de mama moderadamente diferenciado según la clasificación de SBR. Las muestras se recolectaron de Marzo del 2010 a Agosto del 2012 en la UMAE \#25 del IMSS.

Como grupo control se utilizaron 10 muestras de glándula mamaria incluidas en parafina y libres de patología neoplásica o infecciosa.

Análisis Histológico. De las muestras incluidas en bloques de parafina se obtuvieron cortes histológicos de 5 micras que se tiñeron con Hematoxilina y Eosina (H-E) con el fin de corroborar el diagnóstico histopatológico, también se seleccio- naron áreas de tejido adyacentes al tumor para la realización de histoarreglos empleando el dispositivo diseñado por GarcíaGarza et al. (2013).

Posteriormente, de los histoarreglos se obtuvieron cortes histológicos que se tiñeron con el método tricrómico de Masson para diferenciar los componentes epiteliales de los parenquimatosos.

Análisis Histoquímico. Se realizó la reacción histoquímica con azul de toluidina para identificar los gránulos metacromáticos de las células cebadas.

Análisis Inmunohistoquímico. Se emplearon anticuerpos monoclonales específicos anti-receptores hormonales de estrógenos y progesterona (1:200), y anti-Her2/neu (1:50). Para corroborar la presencia de células cebadas observadas en el análisis histoquímico se utilizaron anticuerpos anti-triptasa específica para células cebadas (1:100), y anti-c-Kit (1:600).

Como sistema de detección se utilizó el sistema Envision ${ }^{\circledR}$ (los anticuerpos y el sistema de detección se adquirieron de Dako Cytomationinc $®)$. La positividad se reveló utilizando 3,3'diaminobencidina (DAB). Los núcleos se contrastaron con hematoxilina de Mayer.

Grupos de estudio. Las muestras de glándula mamaria sin patología neoplásica o infecciosa correspondieron al grupo control. Las muestras de adenocarcinoma moderadamente diferenciado se clasificaron en los siguientes grupos de estudio: Grupo A: RH (+) y Her2/neu (+), Grupo B: RH (+) y Her2/ neu (-); y Grupo C: RH (-) y Her2/neu (+). Adicionalmente los grupos se subclasificaron en metastásicos y no metastásicos de acuerdo a la presencia o ausencia de metástasis linfática.

Análisis Morfométrico. En las muestras analizadas con la reacción histoquímica de azul de toluidina, e inmunohistoquímica anti-c-Kit, se cuantificaron las células cebadas positivas/campo de alto poder en 10 campos al azar por cada laminilla analizada. De los resultados se obtuvo el valor promedio y desviación estándar. Los grupos se contrastaron entre sí con la prueba estadística de t de student para identificar diferencias significativas para un valor de $p \leq 0,05$.

\section{RESULTADOS}

De las muestras analizadas el grupo B presentó el mayor porcentaje de frecuencia con un $88 \%$. En cuanto a la presencia de metástasis linfática, las muestras del grupo A no presentaron metástasis, a diferencia de los grupos B y $\mathrm{C}$ que presentaron un $57 \%$ y $66 \%$ de metástasis respectivamente. 
HERNÁNDEZ-ALVIDREZ, O. M.; SOTO-DOMÍNGUEZ, A.; GONZÁLEZ-MURILLO, E. A.; CASTELÁN-MALDONADO, E. E.; GARCÍA-GARZA, D. \& GARCÍA-GARZA, R. Células cebadas y sus patrones de expresión del receptor c-Kit (CD117) como probables indicadores de metástasis en el adenocarcinoma de mama en humanos. Int. J. Morphol., 31 (2):600-605, 2013.

Mediante la reacción histoquímica de azul de toluidina y la inmunohistoquímica anti-triptasa, se observaron células cebadas positivas en todos los grupos de estudio (Fig. 1A y B).

En el análisis morfométrico se observó que en el grupo control existió una escasa cantidad de células cebadas, esta cantidad no se incrementa significativamente en el grupo A. En los grupos B y C se observó un incremento significativo en el número de células cebadas, y fue más evidente en los casos en donde existió metástasis reportada a linfonodos (Fig. 1 C).

Destaca que al analizar los patrones de expresión de c-Kit en las células cebadas peritumorales se observó del patrón membranoso habitual, además de patrones citoplasmáticos difusos y generalizados que se denominaron patrones 1,2 y 3 respectivamente (Fig. 1 D).

Los conteos de dichos patrones de expresión revelaron que en el grupo control se presentan estos tres patrones de expresión. En los grupos A, B sin metástasis y C sin metástasis se observó un predominio el patrón 2, a diferencia de los grupos B y C con metástasis que presentaron en ambos casos un decremento importante de las células cebadas con patrón 2, así como un incremento significativo de los patrones 1 y 3 (Fig. $1 \mathrm{E}$ ).

\section{DISCUSIÓN}

El adenocarcinoma de mama es una neoplasia de gran interés en la actualidad, ya que es el segundo tumor maligno más frecuente en mujeres después del cáncer de pulmón del cual se ha reportado una gran tasa de morbi-mortalidad (Dixon et al.).

En este estudio se observó que la mayoría de los adenocarcinomas moderadamente diferenciados de mama en la población analizada mostró RH (+) y Her2/neu (-). Estos resultados coinciden con lo reportado en otros estudios de adenocarcinoma de mama en humanos (Crabtree $e t$ al., 2005).
La mayor incidencia de metástasis se presentó en el grupo RH (-) y Her2/neu (+), esto ha sido reportado previamente como un factor de mal pronóstico (Khosravi Shahi \& Pérez Manga, 2006).

En este estudio el área de muestreo seleccionada correspondió a la región peritumoral, la cual ha sido denominada como "hot spots", esta región es el sitio donde se concentra una gran cantidad de células cebadas y es ideal para su evaluación (Michailidou et al., 2008).

En nuestras muestras se observó un incremento significativo en el número de células cebadas en los grupos $\mathrm{B}$ y $\mathrm{C}$, este incremento fue más acentuado en los subgrupos que presentaron metástasis a linfonodos axilares.

Otros estudios han reportado la presencia de un mayor número de células cebadas en diversos tumores (Harveit et al., 1984; Dabbous et al., 1986; Ueda et al., 1988) incluyendo al adenocarcinoma de mama, y su incremento se ha asociado a una menor sobrevida.

En algunos estudios se sugiere que las moléculas contenidas en los gránulos de las células cebadas tienen actividad proangiogénica y antigénica (Elpek et al., 2001; Tomita et al., 2001), esto es de suma importancia, ya que la angiogénesis es un paso necesario y crítico para el crecimiento y diseminación del tumor favoreciendo la metástasis (Folkman, 1992).

Al analizar los patrones de expresión de c-Kit en nuestras muestras no se encontraron variaciones relacionadas a la positividad de los RH y Her2/neu, sin embargo se observó que en los subgrupos que presentaron metástasis se invertían los patrones de expresión predominantes.

A la fecha no existen reportes en la literatura que evalúen los patrones de expresión de c-Kit en muestras de adenocarcinoma moderadamente diferenciado de mama, sin embargo un estudio realizado en perros con mastocitoma cutáneo reveló que en los animales en donde predominaba el patrón citoplasmático generalizado presentaban un peor pronóstico debido a la gran proliferación de este tipo celular (Webster et al.).

Fig. 1 A) Microfotografía de adenocarcinoma de mama que muestra células cebadas con gránulos metacromáticos (flechas). Azul de toluidina. Microscopia de luz. 400x. B) Microfotografía de adenocarcinoma de mama que muestra células cebadas positivas para triptasa (flechas). Inmunohistoquímica. Microscopia de luz. 400x. C). Gráfica que muestra los diferentes patrones de expresión de c-Kit en las células cebadas en cada uno de los grupos analizados. D). Microfotografía de adenocarcinoma de mama que muestra los patrones de expresión de c-Kit en las células cebadas: membranoso (flecha blanca), citoplasmático focalizado (flecha roja) y citoplasmático generalizado (flecha azul). Inmunohistoquímica. Microscopia de luz. 400x. E). Gráfica que muestra el promedio de células cebadas/campo en cada uno de los grupos analizados.* $\mathrm{p} \leq 0,05$ 
HERNÁNDEZ-ALVIDREZ, O. M.; SOTO-DOMÍNGUEZ, A.; GONZÁLEZ-MURILLO, E. A.; CASTELÁN-MALDONADO, E. E.; GARCÍA-GARZA, D. \& GARCÍA-GARZA, R. Células cebadas y sus patrones de expresión del receptor c-Kit (CD117) como probables indicadores de metástasis en el adenocarcinoma de mama en humanos. Int. J. Morphol., 31(2):600-605, 2013.
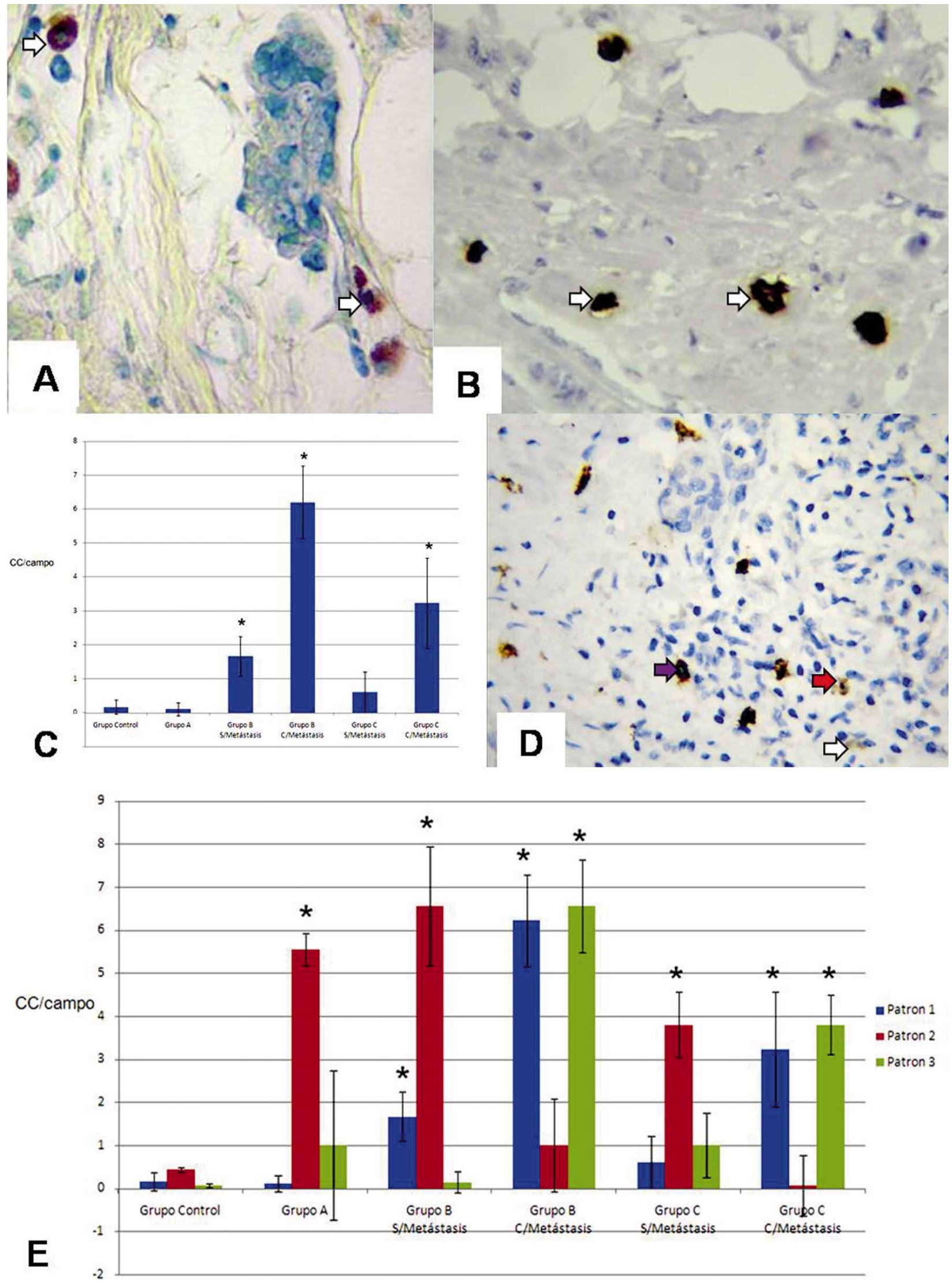
HERNÁNDEZ-ALVIDREZ, O. M.; SOTO-DOMÍNGUEZ, A.; GONZÁLEZ-MURILLO, E. A.; CASTELÁN-MALDONADO, E. E.; GARCÍA-GARZA, D. \& GARCÍA-GARZA, R. Células cebadas y sus patrones de expresión del receptor c-Kit (CD117) como probables indicadores de metástasis en el adenocarcinoma de mama en humanos. Int. J. Morphol., 31 (2):600-605, 2013.

Cabe mencionar que la sobreexpresión de c-Kit identificada por una positividad citoplasmática generalizada está relacionada con un incremento en la tasa de proliferación de células cebadas (Webster et al.). Esto concuerda con los hallazgos observados en los subgrupos metastásicos, que mostraron predominantemente el patrón generalizado y un gran número de células cebadas.

La cantidad de células cebadas por campo, así como las variaciones en cuanto a sus patrones de expresión de c-Kit pueden ser de utilidad en la evaluación integral de las muestras de adenocarcinoma de mama. Esto pudiera ser útil sobre todo en los casos que no se cuente con linfonodos, ya que los parámetros mencionados en este trabajo son indicadores probables, más no definitivos de la presencia de metástasis.
A pesar de no contar con los datos correspondientes a la sobrevida de los pacientes, se ha reportado que la aparición de metástasis está relacionada con un peor pronóstico en un gran número de neoplasias malignas (Weidner et al., 1991; Yoshiji et al., 1996) incluyendo el adenocarcinoma de mama.

\section{AGRADECIMIENTOS}

Los autores agradecemos a la QFB Adriana I. Gallardo-Gómez y al Biól. René Reynaga Piña por su valioso apoyo técnico en la elaboración de este proyecto.

HERNÁNDEZ-ALVIDREZ, O. M.; SOTO-DOMÍNGUEZ, A.; GONZÁLEZ-MURILLO, E. A.; CASTELÁN-MALDONADO, E. E.; GARCÍA-GARZA, D. \& GARCÍA-GARZA, R. Mast cells and expression patterns of c-Kit (CD117) receptor as probable indicators of metastases in human breast adenocarcinoma. Int. J. Morphol., 31(2):600-605, 2013.

SUMMARY: Breast adenocarcinoma is the second most common neoplasm, just after lung cancer in women. According to histopathological features this tumor is classified into well, moderate and poorly differentiated. In addition to this classification, current analysis also use immunohistochemistry to detect estrogen and progesterone receptors (RH), and HER2/neu, this information is useful to make a prognostic in treatment response and survival time. Mast cells have been associated with bad prognosis, because they participate in angiogenesis and tumor growth promoting metastases. This decreases life expectancy. Also mast cells express in the plasmatic membrane the receptor c-Kit (CD117) that is associated to cell proliferation. In this study, we evaluated if there are variations in the expression patterns of c-Kit receptor in mast cells present in breast adenocarcinoma samples; and if expression patterns are associated with the presence of metastases to ganglion. Samples of moderately differentiated adenocarcinoma were evaluated using histological, histochemistry and immunohistochemistry methods. Groups were classified according to their positivity to RH and HER2/neu, and subclassified if presented or not metastases. Also mast cells and expression patterns of c-Kit receptor were analyzed by a morphometric method. Groups with metastases showed higher amount of mast cells, compared with control group and their corresponding group without metastases. Mast cells with expression patterns 1 and 3 were more abundant in all the groups with metastasis. In groups without metastases mast cells with pattern 2 were predominant. Results suggest that the amount of mast cells and the expression patterns of c-Kit could be use as probable indicators, but not definitive of the presence of nodal metastases.

KEY WORDS: Breast adenocarcinoma; Metastases; Mast cells.

\section{REFERENCIAS BIBLIOGRÁFICAS}

Allred, D. C.; Harvey, J. M.; Berardo, M. \& Clark, G. M. Prognostic and predictive factors in breast cancer by immunohistochemical analysis. Mod. Pathol., 11(2):155-68, 1998.

Amat, S.; Penault-Llorca, F.; Cure, H.; Le Bouedëc, G.; Achard, J. L.; Van Praagh, I. et al., Scarff-Bloom-Richardson (SBR) grading: a pleiotropic marker of chemosensitivity in invasive ductal breast carcinomas treated by neoadjuvant chemotherapy. Int. J. Oncol., 20(4):791-6, 2002.

Bilous, M.; Dowsett, M.; Hanna, W.; Isola, J.; Lebeau, A.; Moreno, A.; et al. Current perspectives on HER2 testing: a review of national testing guidelines. Mod. Pathol., 16(2):173-82, 2003.
Columbo, M.; Horowitz, E. M.; Botana, L. M.; MacGlashan, D. W. Jr.; Bochner, B. S.; Gillis, S. et al. The human recombinant c-kit receptor ligand, rhSCF, induces mediator release from human cutaneous mast cells and enhances IgE-dependent mediator release from both skin mast cells and peripheral blood basophils. J. Immunol., 149(2):599-608, 1992.

Crabtree, R.; Neme, Y.; Rivera, R. \& Olivares, B. Receptores hormonales, HER-2/neu y p53 en pacientes con cáncer de mama. GAMO, 4(2):29-33, 2005.

Dabbous, M. K.; Walker, R.; Haney, L.; Carter, L. M.; Nicolson, G. L. \& Woolley, D. E. Mast cells and matrix degradation at sites of tumour invasion in rat mammary adenocarcinoma. $\mathrm{Br}$. 
HERNÁNDEZ-ALVIDREZ, O. M.; SOTO-DOMÍNGUEZ, A.; GONZÁLEZ-MURILLO, E. A.; CASTELÁN-MALDONADO, E. E.; GARCÍA-GARZA, D. \& GARCÍA-GARZA, R. Células cebadas y sus patrones de expresión del receptor c-Kit (CD117) como probables indicadores de metástasis en el adenocarcinoma de mama en humanos. Int. J. Morphol., 31(2):600-605, 2013.

\section{J. Cancer, 54(3):459-65, 1986.}

Dixon, J. M.; Page, D. L.; Anderson, T. J.; Lee, D.; Elton, R. A.; Stewart, H. J. et al. Long-term survivors after breast cancer. Br. J. Surg., 72(6):445-8, 1985.

Elledge, R. M.; Green, S.; Pugh, R.; Allred, D. C.; Clark, G. M.; Hill, J. et al. Estrogen receptor (ER) and progesterone receptor (PgR), by ligand-binding assay compared with ER, $\mathrm{PgR}$ and $\mathrm{pS} 2$, by immuno-histochemistry in predicting response to tamoxifen in metastatic breast cancer: a Southwest Oncology Group Study. Int. J. Cancer, 89(2):111$7,2000$.

Elston, C. W. \& Ellis, I. O. Pathological prognostic factors in breast cancer. I. The value of histological grade in breast cancer: experience from a large study with long-term follow-up. Histopathology, 19(5):403-10, 1991.

Elpek, G.; Gelen, T.; Aksoy, N. H.; Erdogan, A.; Dertsiz, L.; Demircan, A. et al. The prognostic relevance of angiogenesis and mast cells in squamous cell carcinoma of the oesophagus. J. Clin. Pathol., 54(12):940-4, 2001.

Folkman, J. The role of angiogenesis in tumor growth. Semin. Cancer Biol., 3(2):65-71, 1992.

García-Closas, M.; Hall, P.; Nevanlinna, H.; Pooley, K.; Morrison, J.; Richesson, D. A. et al. Heterogeneity of breast cancer associations with five susceptibility loci by clinical and pathological characteristics. PLoS Genet., 4(4):e1000054, 2008 .

García-Garza, R.; Rodríguez-Vidales. E. P. \& Soto-Domínguez, A. Quick and inexpensive method to elaborate tissue punches useful in paraffin tissue microarrays. Int. J. Morphol., 31(1):504, 2013.

Harvey, J. M.; Clark, G. M.; Osborne, C. K. \& Allred, D. C. Estrogen receptor status by immunohistochemistry is superior to the ligand-binding assay for predicting response to adjuvant endocrine therapy in breast cancer. J. Clin. Oncol., 17(5):1474$81,1999$.

Hartveit, F.; Thoresen, S.; Tangen, M. \& Maartmann-Moe, H. Mast cell changes and tumour dissemination in human breast carcinoma. Invasion Metastasis, 4(3):146-55, 1984.

Kitamura, Y.; Kanakura, Y.; Sonoda, S.; Asai, H. \& Nakano, T. Mutual phenotypic changes between connective tissue type and mucosal mast cells. Int. Arch. Allergy Appl. Immunol., 82(34):244-8, 1987.

Khosravi Shahi, P. \& Pérez Manga, G. The clinical relevance of the overexpression of HER-2 in breast cancer. An. Med. Interna, 23(3):103-4, 2006.

Metcalfe, D. D.; Baram, D. \& Mekori, Y. A. Mast cells. Physiol. Rev., 77(4):1033-79, 1997.
Michailidou, E. Z.; Markopoulos, A. K. \& Antoniades, D. Z. Mast cells and angiogenesis in oral malignant and premalignant lesions. Open Dent. J., 2:126-32, 2008

Mohisin, S. K.; Weiss, H.; Havighurst, T.; Clark, G. M.; Bernardo, M.; Roanh, le D. et al. Progesterone receptor by immunohistochemistry and clinical outcome in breast cancer: a validation study. Mod. Pathol., 17(12):1545-54, 2004.

Norrby, K. \& Sörbo, J. Heparin enhances angiogenesis by a systemic mode of action. Int. J. Exp. Pathol., 73(2):147-55, 1992.

Philips, T.; Murray, G.; Wakamiya, K.; Askaa, J.; Huang, D.; Welcher, R. et al. Development of standard estrogen and progesterone receptor inmunohistochemical assays for selection of patients for antihormonal therapy. Appl. Immunohistochem. Mol. Morphol., 15(3):325-31, 2007.

Ueda, T.; Aozasa, K.; Tsujimoto, M.; Yoshikawa, H.; Kato, T.; Ono, K. et al. Prognostic significance of mast cells in soft tissue sarcoma. Cancer, 62(11):2416-9, 1988.

Tomita, M.; Matsuzaki, Y.; Edagawa, M.; Shimizu, T.; Hara, M.; Sekiya, R. et al. Association of mast cells with tumor angiogenesis in esophageal squamous cell carcinoma. Dis. Esophagus, 14(2):135-8, 2001.

Webster, J. D.; Kiupel, M.; Kaneene, J. B.; Miller, R. \& YuzbasiyanGurkan, V. The use of KIT and tryptase expression patterns as prognostic tools for canine cutaneous mast cell tumors. Vet. Pathol., 41(4):371-7, 2004.

Weidner, N.; Semple, J. P.; Welch, W. R. \& Folkman, J. Tumor angiogenesis and metastasis--correlation in invasive breast carcinoma. N. Engl. J. Med., 324(1):1-8, 1991.

Yoshiji, H.; Gomez, D. E.; Shibuya, M. \& Thorgeirsson, U. P. Expression of vascular endothelial growth factor, its receptor, and other angiogenic factors in human breast cancer. Cancer Res., 56(9):2013-6, 1996.

Correspondence to:

Dr. Rubén García Garza

Facultad de Medicina de la UANL

Departamento de Histología, AP 1563

Monterrey, N. L. 64400

MÉXICO

Tel. 52-81-8329-4000 Ext. 2685

Email: rubengarcia85@hotmail.com

Recibido : 19-12-2012

Aceptado: 02-03-2013 